\title{
Lässt sich konzentrierte städtische Armut überwinden?
}

\author{
Erfahrungen aus den USA
}

Vorbemerkung: Zu den letzten Projekten, die Hartmut Häußermann mit großem Nachdruck verfolgt hat, gehört die Auswertung der Erfahrungen mit Politikansätzen in den USA, die auf eine Verringerung der sozialräumlichen Konzentration von Armut in den Großstädten abzielen. Er hat sich diese Erfahrungen nicht nur über Literatur, sondern auch durch eigenen Augenschein während einer USA-Reise und durch Gespräche mit Kolleginnen und Kollegen angeeignet. Für das Bundesministerium für Verkehr, Bau und Stadtentwicklung, das seine Arbeit gefördert hat, verfasste er ein Gutachten unter dem Titel »Moving to Opportunity (MTO). Eine Chance zur Weiterentwicklung der Städtebauförderung " (Oktober 2010). Ich hatte mich meinerseits mit dem Pendant zu MTO, der Neuorientierung von »Public Housing " mit dem Programm HOPE VI, während eines Forschungssemesters im Sommer 2011 beschäftigt. Geplant war, die Erkenntnisse aus beiden Recherchen in ein gemeinsames größeres Projekt einfließen zu lassen, zu dem es nun leider nicht mehr kommt. Gelegentlich übernahm in der Kooperation zwischen uns einer von beiden die »Führung « in der Weise, dass er Textvorlagen des anderen mit eigenen Texten zu einem eigenständigen Beitrag verband. $\mathrm{Zu}$ diesem Verfahren habe ich auch diesmal gegriffen. Aus der unveröffentlichten Kurzfassung des Berichts für das Ministerium habe ich längere Passagen zur Evaluation von MTO und den Folgerungen aus MTO wörtlich oder inhaltlich übernommen, so dass ein gemeinsamer Text entstand, wenn auch keine gemeinsame Endredaktion mehr möglich war. M.K. ${ }^{1}$

\section{Konzentrierte städtische Armut - das Problem}

Eine hohe Konzentration von Armen und Arbeitslosen in bestimmten Quartieren fügt den Menschen mit geringem Einkommen, die dort leben, noch zusätzliche Benachteiligungen hinzu. Diese These von den »Quartierseffekten « ist mittlerweile in der internationalen Forschung immer wieder bestätigt worden, wenngleich sich die Intensität und Ausprägung der Effekte in den verschiedenen Ländern und Städten, ja sogar zwischen Vierteln innerhalb einer Stadt unterscheiden. Sozialbeziehungen, die sich auf Menschen in ähnlich benachteiligter Lage im Quartier kon-

1 Dem Bundesministerium für Verkehr, Bau und Stadtentwicklung danke ich für die Genehmigung, die Kurzfassung des Berichts in diesen Aufsatz einzuarbeiten. Für hilfreiche Kommentare danke ich Wulf Hopf, Günther Schmid, Uwe-Jens Walther, Jens Wurtzbacher sowie Reinhard Blomert und den Gutachtern des Leviathan. 
zentrieren, schneiden Erwachsene von Kontakten ab, die am Arbeitsmarkt weiterhelfen könnten. Eine »schlechte Adresse « behindert die Arbeitssuche zusätzlich. Für Jugendliche aus armen Familien, die unter sich bleiben, kann die Nachbarschaft zum negativen Lernraum werden, der sie weiter abdriften lässt von Schule und Ausbildung. Die Qualität der Schulen und anderer Einrichtungen der lokalen Infrastruktur ist häufig schlechter als in wohlhabenderen Vierteln. Unsicherheit im öffentlichen Raum verstärkt das Gefühl der sozialen Isolation und erschwert die Bewältigung des Alltags. Gleichwohl bieten solche Quartiere auch einen gewissen sozialen Schutz. Solange man sich unter seinesgleichen bewegt, kommt es weniger zur Konfrontation mit kritischen, weil diskriminierenden Situationen mit der "Außenwelt «. Und enge Kontakte untereinander führen zwar nicht aus den bedrückenden Verhältnissen heraus, aber sie leisten zumindest vielfältige Hilfen. ${ }^{2}$

Seit den 1980er Jahren lieferten Studien aus den USA, vor allem aus Chicago, dramatische Befunde über die Formierung einer neuen städtischen "underclass ", bedingt durch eine hohe sozialräumliche Konzentration von Armut. ${ }^{3}$ Die europäische Stadt- und Ungleichheitsforschung zog diese Befunde immer wieder zum Vergleich heran. Dabei zeigte sich, dass die Wirkmechanismen an den Arbeitsmärkten ähnlich, aber die Folgen verschieden sind, da die europäischen Sozialstaaten die Armutskonzentration und die Nachbarschaftseffekte in der Regel abschwächten. Andererseits galten die amerikanischen Städte aber auch gewissermaßen als Menetekel für den Fall, dass die europäischen Staaten und Gesellschaften noch weiter in die Richtung einer "Amerikanisierung " ihrer Institutionen einschwenken sollten.

Nun fand allerdings in den letzten beiden Jahrzehnten in den USA eine Reihe bemerkenswerter Entwicklungen statt, die bisher in der deutschen Stadt- und Ungleichheitsforschung kaum zur Kenntnis genommen worden sind. Während der 1990er Jahre ging die konzentrierte Armut in den meisten amerikanischen Metropolenregionen deutlich zurück. Zugleich erfolgte auf Bundesebene eine politische Neuorientierung, die sich dezidiert die Reduzierung konzentrierter Armut mit Hilfe verschiedener Programme zum Ziel setzte. Der Wiederanstieg der Armut seit der Jahrtausendwende und insbesondere nach dem tiefen Kriseneinschnitt von 2008 wirft die Frage auf, ob erneut mit einer Zunahme der räumlichen Konzentration städtischer Armut zu rechnen ist.

Das Ziel der folgenden Ausführungen ist es zunächst, die Entwicklung der Armut und ihrer sozialräumlichen Verteilung in den amerikanischen Metropolenregionen während der zurückliegenden beiden Jahrzehnte nachzuzeichnen, um die Veränderungen, die stattgefunden haben, zu verdeutlichen. Die Untersuchung der Gründe für diese Veränderungen ist nicht abgeschlossen, aber es ist möglich, aus dem aktuellen Diskussionsstand ein vorläufiges Fazit zu ziehen. Dabei zeigt sich insbesondere der Einfluss von Arbeitsmarktlage, Einkommensungleichheit und Migrationsbewegungen auf die räumliche Verdichtung von Armut.

Sodann werden wir auf die bundespolitische Wendung in den USA eingehen, die »tiefer", nämlich an den Quartieren selbst ansetzt und dadurch zur Dekonzentra-

2 Vgl. Häußermann, Kronauer 2009, S. 163-171; Kronauer 2008.

3 Richtungsweisend Wilson 1987. 
tion der Armut beitragen will. Das Augenmerk liegt dabei einerseits auf der Neuausrichtung von »Public Housing " im Rahmen des Programms HOPE VI, andererseits auf der Evaluierung eines speziellen Programms zur Förderung der Abwanderung aus Nachbarschaften mit konzentrierter Armut, MTO. Beide Programme stehen in enger Verbindung zueinander. Sie erlauben es, "quasi-experimentell « Möglichkeiten und Grenzen einer ausschließlich auf Quartierseffekte abzielenden Politik zur Armutsverminderung zu überprüfen. Abschließend folgen einige Überlegungen darüber, welche Lehren auch für die deutsche/europäische Diskussion über Armut und Quartierseffekte von Bedeutung sein könnten.

\section{Veränderungen in der städtischen Armut der USA}

Von Mitte der 1970er Jahre bis Mitte der 1990er Jahre hatte die Armut, gemessen an der offiziellen Armutsgrenze, in den USA stetig zugenommen. Danach kam es zu einer Wende in der Armutsentwicklung, die bis 2000 anhielt. ${ }^{4}$ Die absolute, an jener Grenze bestimmte Armut ging zurück, allerdings bei gleichzeitigem Anstieg der relativen (auf das Durchschnittseinkommen bezogenen) Armut. ${ }^{5}$ Zum ersten Mal seit den 1970er Jahren verzeichneten die unteren Einkommensgruppen in den USA gegenüber den mittleren Einkommen leichte Zugewinne, während die Ungleichheit zwischen Einkommensmitte und oberen Einkommen weiter zunahm. ${ }^{6}$ Die Verbindung von Wirtschafts- und Beschäftigungswachstum hatte günstige Arbeitsmarktbedingungen auch für gering qualifizierte Arbeitskräfte geschaffen. Dazu wirkte die Ausweitung der negativen Einkommenssteuer (Earned Income Tax Credit ${ }^{7}$ ) auf weitere berechtigte Gruppen als wichtige armutsreduzierende Finanzhilfe für die »working poor ", aber auch umgekehrt als staatliche Subvention von Niedriglöhnen. ${ }^{8}$ Gleichzeitig verschärfte sich allerdings die Tendenz zur Abkoppelung bestimmter Gruppen, vor allem junger Afro-Amerikaner, vom Arbeitsmarkt. Die in den Arbeitslosenstatistiken nicht aufgeführte Gefängnispopulation nahm zu. ${ }^{9} \mathrm{Zu}$ den problematischen Seiten gehörte auch, dass bereits seit Mitte der 1990er Jahre das amerikanische Beschäftigungswachstum in einem erheblichen Maße auf Sand, sprich: eine wachsende Verschuldung der privaten Haushalte gebaut war. Diese

4 Vgl. Mishel et al. 2005, S. 313.

5 Vgl. Iceland 2006, S. $104 \mathrm{f}$.

6 Vgl. Mishel et al. 2005, S. 64-68.

7 Der Earned Income Tax Credit (EITC) ist eine Form der steuerlichen Unterstützung der Bezieher niedriger Erwerbseinkommen. Berechtigte mit einem Jahreseinkommen unterhalb der Steuerpflicht erhalten ihrerseits Geld aus Steuermitteln, das ihr Einkommen innerhalb einer bestimmten Bandbreite und je nach Einkommenshöhe abgestuft aufstockt. 1975 eingeführt, war der EITC ursprünglich vor allem für Erwerbstätige mit Kindern gedacht. Die Clinton-Administration erhöhte 1993 nicht nur die Bezüge deutlich, sondern erweiterte auch den Kreis der Bezugsberechtigten auf Erwerbstätige ohne Kinder (vgl. Katz 2001, S. 294 f.).

8 Vgl. Mishel et al. 2009, S. 71 f., 299; Katz 2001, S. 316.

9 Vgl. Katz, Stern 2006, S. 88 f.

Leviathan, 40. Jg., 3/2012 
Problematik spitzte sich im folgenden Jahrzehnt mit der von Politik und Finanzmärkten erzeugten Immobilienblase weiter zu.

Nach 2000 folgte auf Stagnation ein erneuter Rückschritt. Zunächst stieg die Armut trotz zusätzlicher Produktivitätsgewinne in der Ökonomie wieder leicht an. ${ }^{10} \mathrm{Im}$ Anschluss an die Finanz- und Wirtschaftskrise von 2008 nahm sie dann drastisch zu. Laut Bericht des Census Bureau erreichte sie 2010 wieder das Niveau von 1993, nämlich 15,1 Prozent gemessen an der offiziellen Armutsgrenze. ${ }^{11}$ Das mittlere Familieneinkommen ging gegenüber den späten 1990er Jahren zurück, die Einkommen im unteren und oberen Einkommensbereich drifteten weiter auseinander. »Dies ist wirklich ein verlorenes Jahrzehnt «, beklagte Bruce Katz von der Brookings Institution. ${ }^{12}$

Wie manifestiert sich das Auf und Ab der Armutsraten in den Metropolenregionen des Landes? Von Mitte der 1970er bis in die frühen 1990er Jahre hatten die Armut und ihre räumliche Konzentration in den Kernstädten der Metropolengebiete stetig zugenommen. Nachbarschaften (statistisch erfasst als »census tracts «) mit Anteilen von mindestens 40 Prozent an armen Bewohnerinnen und Bewohnern breiteten sich aus. In die 1980er Jahre fällt die Diagnose der Entstehung einer städtischen » underclass «, hervorgerufen durch die soziale Isolation der Menschen, die unter den Bedingungen konzentrierter Armut leben müssen.

Zwischen 1990 und 2000 jedoch schien dieser Trend gebrochen. Es war ein leichter Rückgang der Armut in den Kernstädten der großen Metropolengebiete zu verzeichnen, vor allem aber ein deutlicher Rückgang der Viertel mit sehr hohen Anteilen von Armen (Armutsquoten von 40 Prozent und mehr) in den Kernstädten sowie der in solchen Vierteln lebenden Bewohner. ${ }^{13}$ Jargowsky und Yang diagnostizierten für diese Periode ebenfalls einen Rückgang der »underclass «. ${ }^{14}$ Wermutstropfen in dieser positiven Bilanz (» hidden dangers «, Jargowsky) bildeten allerdings die ersten Anzeichen einer "Suburbanisierung « der Armut. ${ }^{15}$ Darüber hinaus bedarf die Feststellung eines Rückgangs von Armut und konzentrierter Armut in den Kernstädten jedoch noch weiterer Einschränkungen und Differenzierungen.

Sowohl was die Entwicklung der Armut insgesamt als auch die der konzentrierten Armut betrifft, bestanden in der Dekade von 1990 bis 2000 erhebliche regionale

10 Vgl. Mishel et al. 2009, S. 125, 301.

11 Vgl. DeNavas-Walt et al. 2011.

12 Zitiert in der New York Times, 14. September 2011, S. A1.

13 Der Rückgang der Bevölkerung in Gebieten mit sehr hohen Konzentrationen von Armut betrug laut Jargowsky insgesamt 24 Prozent, in den Kernstädten 21 Prozent. Vgl. Jargowsky 2003, S. 12. Dreier et al. weisen allerdings darauf hin, dass die Bevölkerung in Nachbarschaften (»census tracts «) mit Armutsanteilen von 30 bis 40 Prozent gleich geblieben sei (2004, S. 56), der Rückgang konzentrierter Armut also bescheidener ausfällt, als es Jargowskys Angaben nahelegen.

14 Vgl. Jargowsky, Yang 2006.

15 Gemeint sind damit eine Verringerung der Armut in den Kernstädten der Metropolenregionen sowie eine Zunahme der Armut in den angrenzenden Gebieten innerhalb der Metropolenregionen. Vgl. Jargowsky 2003, S. 11-13; Berube, Frey 2005. 
Unterschiede. Entgegen dem Trend in anderen Regionen nahmen beide in den Kernstädten der Metropolengebiete der Westküste zu, eine Folge der Rezession der frühen 1990er Jahre und der starken Zuwanderung aus Süd- und Zentralamerika.

Gravierende Unterschiede fanden sich aber auch auf der Quartiersebene, wie eine Studie des Urban Institute zeigt. Es gab sowohl Aufstiege aus konzentrierter Armut (hier allerdings definiert anhand einer Armutsquote von 30 Prozent) als auch Abstiege in konzentrierte Armut. Dabei überwog selbst in den 1990er Jahren noch immer die Zahl der Abstiege (Verschlechterungen der Armutssituation von »census tracts «/Nachbarschaften), jedoch in geringerem Maße als im Jahrzehnt zuvor. Die Verschlechterungen betrafen vor allem Quartiere in den inneren suburbanen Ringen und solche mit starker Zuwanderung von Minderheiten, insbesondere von »Hispanics «. ${ }^{16}$ Und schließlich: In den 1990er Jahren nahmen die Segregation nach Einkommen (auch innerhalb der »rassischen $«$ Kategorien ${ }^{17}$ ) sowie die rassische Segregation der Schuldistrikte weiter zu. ${ }^{18}$

Trotz dieser Relativierungen gilt es für die 1990er Jahre festzuhalten, dass es zu einem, wenn auch uneinheitlichen, Rückgang der Armut und der konzentrierten Armut in den meisten Metropolenregionen gekommen war. Als Gründe hierfür gelten zuallererst die bereits angesprochenen Faktoren der allgemeinen Arbeitsmarktentwicklung in Verbindung mit einer Anhebung des Mindestlohns und der Lohnsubvention im Niedriglohnbereich durch den Earned Income Tax Credit. Hingegen fanden die Ökonomen Partridge und Rickman bei einem Vergleich der Bundesstaaten kaum Belege für einen Einfluss der »welfare reform «19 von 1996 auf die Verringerung von Armut. ${ }^{20}$

16 Vgl. Kingsley, Pettit 2007.

17 » Rassisch « wird hier und im Folgenden im Sinne des amerikanischen » race « gebraucht, wie es im Zensus und anderen statistischen Erhebungen verwendet wird, mit der Unterteilung »White«, »Non-White Hispanic«, "African American« (oder »Black«) und $»$ Asian $\ll$.

18 Vgl. Briggs 2005, S. 28, 32.

19 Die während der Präsidentschaft von Bill Clinton mit einer Mehrheit von Demokraten und Republikanern beschlossene Reform beendete das bis dahin wichtigste, auf Familien zugeschnittene Hilfsprogramm Aid to Families with Dependend Childen (AFDC), etablierte stattdessen zwei Hilfsprogramme mit Bundesmitteln, aber in der Verantwortung der Bundesstaaten, darunter Temporary Assistance for Needy Families (TANF), beschränkte das Recht auf Unterstützung durch dieses Programm auf fünf Jahre während der Lebenszeit (erlaubte allerdings den Bundesstaaten Abweichungen von der Regel), entzog neu Eingewanderten das Recht auf Unterstützung und beschränkte die ohnehin sehr geringen Ansprüche auf Unterstützungsleistungen für arbeitsfähige Erwachsene ohne Kinder noch weiter (vgl. Katz 2001, S. 303 f., 317-328). Alle diese Maßnahmen koppelten Sozialleistungen noch enger an eine individuelle Pflicht zur Erwerbsarbeit. Die Bundesstaaten sollten Kinderbetreuungsangebote und Qualifizierungsprogramme bereitstellen, um alleinerziehende Mütter zu befähigen, Erwerbsarbeit anzunehmen. Je nach politischer Ausrichtung der Bundesstaaten variiert die Umsetzung der »welfare reform « erheblich.

20 Vgl. Patridge, Rickman 2006, S. 269 f.

Leviathan, 40. Jg., 3/2012 
Höhe und sozialräumliche Verteilung der Armut werden jedoch auch von Binnenmigration und transnationaler Migration beeinflusst. Darüber bestehen kaum Zweifel, wohl aber über die Richtung der Effekte. In den 1990er Jahren verstärkte sich noch einmal deutlich die bereits in den 1970er Jahren einsetzende Abwanderung von "African Americans « in den amerikanischen Süden. Per saldo verlieren inzwischen der Nordosten, Westen und Mittlere Westen der USA, in besonderem Maß aber New York Bewohner dieser Bevölkerungsgruppe. Allerdings ist die Abwanderungsbewegung bei qualifizierten Arbeitskräften stärker ausgeprägt als bei gering qualifizierten Arbeitskräften, so dass Rückschlüsse auf die regionalen oder gar lokalen Folgen für die Verteilung von Einkommen schwierig sind. ${ }^{21}$ Die starke transnationale Zuwanderung von »Hispanics « und »Asians « vergrößerte während der 1990er Jahre die ethnische Diversität der städtischen Nachbarschaften, jedoch wiederum selektiv - »Hispanics « leben mehr mit »African Americans ", "Asians « mehr mit » Whites « zusammen. ${ }^{22}$ Inzwischen sind die Vorstädte zur ersten Anlaufstelle vieler dieser neuen Migranten geworden. ${ }^{23}$ Das erhöht auch dort die ethnische/ rassische Diversität der Quartiere, trägt aber gleichzeitig zum Ansteigen der suburbanen Armut bei. ${ }^{24}$

Wenig ist bekannt über die Mobilitätsmuster in den Armutsgebieten selbst. Eine Studie des Urban Institute weist auf eine hohe Mobilität in solchen Quartieren und dabei auf zwei unterschiedliche Varianten hin: Aufstiegsmobilität aus der Armutsnachbarschaft heraus (geringfügiger als das zweite Muster), überwiegend ökonomisch oder sozial erzwungene Mobilität (»churning «) innerhalb des Viertels oder in angrenzende Gebiete. ${ }^{25}$ Dies legt die Vermutung nahe, dass konzentrierte Armut in manchen Gebieten nur deshalb und nur marginal zurückging, weil Teile der Armutsbevölkerung in benachbarte Quartiere auswichen oder besser gestellte Zugezogene die Einkommensverteilung veränderten. Nicht jeder Rückgang der Armut in einem Quartier muss somit auch bedeuten, dass es den von Armut betroffenen Individuen, die dort wohnen oder gewohnt haben, nun besser geht. Das Problem der »Verschiebung « armer Haushalte aus Vierteln mit sehr hoher Armutsrate in Viertel mit niedrigerer, aber immer noch hoher Armut, ohne dass sich dabei die soziale Lage der Haushalte ändert, zeigt sich auch in den Folgen der Umsiedlungsmaßnahmen, die seit 1992 Teil der Bundesprogramme HOPE VI und MTO zur Bekämpfung konzentrierter städtischer Armut sind. Dazu weiter unten (Kapitel 6) mehr.

Wie sich das »verlorene Jahrzehnt " auf die städtische Armut ausgewirkt hat und weiter auswirkt, ist noch nicht erschöpfend dokumentiert und interpretiert. Die Daten des Zensus von 2010 werden gerade erst ausgewertet. Zwei Entwicklungen zeichnen sich jedoch schon ab: Die Armutsraten stiegen bereits zwischen 2000 und 2008 in den Kernstädten der größten Metropolengebiete des Mittleren Westens,

21 Vgl. Frey 2005.

22 Vgl. Fasenfest et al. 2006.

23 Vgl. Briggs 2005, S. 25.

24 Vgl. Berube, Frey 2005, S. 215, 220; Fortuny et al. 2010.

25 Coulton et al. 2009. 
des Südens und des Westens wieder an, allerdings nicht in denen des Nordostens. Vor allem aber fand eine deutliche Fortsetzung des Trends zur "Suburbanisierung " der Armut in drei der vier Regionen statt. ${ }^{26}$ Mittlerweile leben zum ersten Mal mehr Arme in den "suburbs" als in den Kernstädten der großen Metropolenregionen. Allerdings liegt die Armutsquote in den Vorstädten wegen der größeren Zahl ihrer Bewohner noch immer erheblich unter (bei etwa der Hälfte) der Quote der Kernstädte. ${ }^{27}$ Aufgrund der längerfristigen Verlagerungen von Arbeitsplätzen und Wohnungen in das Umland der Kernstädte macht sich die Krise inzwischen gerade dort in steigenden Armutsraten bemerkbar. Das gilt vor allem in den Metropolenregionen des Mittleren Westens, die den weiteren Niedergang ihrer (Automobil-)Industrie zu verkraften haben.

\section{Die Entwicklung der Armut und ihrer räumlichen Verteilung in den Metropolenregionen - eine Zwischenbilanz}

Die Schwankungen in der Entwicklung der Armutsraten insgesamt ebenso wie die der Armut in den Metropolenregionen über die letzten beiden Jahrzehnte in den USA weisen vor allem auf den starken Einfluss hin, den die wechselnden Arbeitsmarktchancen für gering Qualifizierte auf das Einkommenswachstum und die mehr oder weniger umverteilenden Wirkungen des Sozialstaats auf die Ungleichheit der Einkommen ausüben. Einkommenswachstum hat in den 1990er Jahren die offizielle Armut gesenkt, Einkommensungleichheit die relative Armut jedoch steigen lassen. ${ }^{28}$ Zwischen 2000 und 2006 überwogen die negativen Effekte der sich noch weiter vergrößernden Einkommensungleichheit auch die positiven Effekte des Wirtschaftswachstums, so dass bereits vor der großen Krise selbst die offizielle Armut wieder anstieg. ${ }^{29}$ Die Befunde werfen ein scharfes Licht auf die zurückliegende amerikanische Debatte über die »underclass «. Sie widersprechen deutlich den Behauptungen der konservativen Sozialstaatsgegner, weniger die fehlenden Arbeitsmarktchancen und die ungleiche Einkommensverteilung als eine verfehlte moralische Haltung und die "Kultur der Armut « bei den Betroffenen seien für die hohe innerstädtische Armut verantwortlich. ${ }^{30}$

Bei der sozialräumlichen Verteilung der Armut spielt überdies die in den USA stark ausgeprägte, von Arbeitsmärkten und Wohnungsmärkten abhängige Binnen- und transnationale Migration eine wichtige Rolle. Die Wirkung der »welfare reform « von 1996 auf die vorübergehende Reduzierung der offiziellen Armutsquote wird dagegen eher gering eingeschätzt. Jedoch dürfte sich die Mischung von unterstüt-

26 Vgl. Kneebone, Garr 2010, die auch für die vierte Region, den Westen, mit einer Zunahme der Armut in den Vorstädten rechnen.

27 Ebd., S. 5, 6.

28 Vgl. Iceland 2006, S. 106-109, 116.

29 Vgl. Mishel et al. 2009, S. 321.

30 Für einen Überblick über die Debatte Kronauer 2010, S. 56-58. 
zenden und repressiven Maßnahmen ${ }^{31}$ auf die interne Zusammensetzung der Armutspopulation und die Verteilung und Zuspitzung der Ausgrenzungsrisiken ausgewirkt haben. So ging in den 1990er Jahren die offizielle Armut (nicht allerdings die relative) von Familien mit alleinerziehenden Müttern deutlich zurück. ${ }^{32}$ Gleichzeitig stieg die Einkerkerungsrate junger afro-amerikanischer Männer an.

Arbeitsmarktentwicklung, sozialstaatliche Umverteilung und Wanderungsbewegungen wirken sich im amerikanischen Beispiel erheblich auf Armutsraten und soziale Zusammensetzung städtischer und suburbaner Quartiere aus. Sie sind aber auf der Quartiersebene ihrerseits kaum beeinflussbar. Gleichwohl bleibt eine auf sozialräumliche Dekonzentration von Armut abzielende Politik auch auf Quartiersebene sinnvoll und möglich. Einen Versuch in dieser Richtung bedeuten die Programme HOPE VI und MTO der amerikanischen Bundesregierung.

\section{Politische Ansätze zur Dekonzentration der städtischen Armut}

HOPE VI (»Homeownership and Opportunity for People Everywhere«) und MTO (»Moving to Opportunity «), beide auf den Weg gebracht 1992/1993, bedeuteten eine grundlegende Neuausrichtung der Wohnungspolitik des Bundes und der dafür zuständigen Behörde für Housing and Urban Development (HUD). Sie stellen erste explizite Ansätze zur Reduzierung von benachteiligenden Quartierseffekten durch die Auflösung sozialräumlicher Konzentrationen von Armut und die gezielte Stärkung von sozialräumlicher Mischung dar.

»Public Housing « war ursprünglich 1937 als Bestandteil des New Deal durchaus auch für Familien mit niedrigem Einkommen und nicht nur für ausgesprochen Arme gedacht. Allerdings wollte die Kongressmehrheit von Anfang an verhindern, dass der öffentliche Wohnungssektor in Konkurrenz zum privaten Immobilienmarkt tritt. Dementsprechend fiel die beschränkte finanzielle Ausstattung des Programms aus, mit der Folge einer chronischen Unterfinanzierung der Projekte. Die billige und vom Design her isolierende und abstoßende Bauweise separierte die Anlagen bereits physisch von den sie umgebenden Nachbarschaften. Wer es sich nach dem Krieg leisten konnte, wegzuziehen, verließ die »Projects «, die mehr und mehr zu Behausungen der Armen wurden. ${ }^{33}$ Schwartz betont allerdings, dass trotz der beschränkten Mittel, aufsehenerregender Fälle von Missmanagement (notorisch: Chicago) und trotz des schlechten Rufs vieler Projekte die meisten »Public Housing Authorities « (vorbildlich: New York) gute Arbeit leisten und anständigen Wohnraum bereitstellen. ${ }^{34}$

HOPE VI setzt vor allem auf die Änderung der physischen Infrastruktur (Renovierung oder Abriss der am meisten physisch und sozial beeinträchtigenden Wohnanlagen), aber auch auf eine stärkere soziale Mischung in den an Stelle der abgeris-

31 Siehe Fußnote 19.

32 Vgl. Iceland 2006, S. 104.

33 Schwartz 2010, S. 129, $134 \mathrm{f}$.

34 Ebd., S. 125, 136, $142 \mathrm{f}$. 
senen Projekte errichteten neuen Einheiten von »Public Housing «35 sowie die Ermöglichung von Mobilität durch Wohnberechtigungsscheine (»vouchers «). ${ }^{36}$ Gleichzeitig sollen durch die Einführung neuer Finanzierungsmodelle auch die private Wohnungswirtschaft (beispielsweise mit Hilfe steuerlicher Förderung von privatem Wohnungsangebot für Mieter mit niedrigem Einkommen) und Nicht-Regierungsorganisationen ins Boot geholt, soll der Dualismus von »Public Housing « und privater Immobilienwirtschaft also überwunden werden. Darüber hinaus wird die Verwaltung der Projekte dezentralisiert und den Managementmethoden in der privaten Immobilienindustrie angepasst. Die architektonischen und stadtplanerischen Konzepte für die Neuerrichtung und Rehabilitation der Gebäude sind Ideen des "New Urbanism « verpflichtet, einer Bebauungsdichte im »menschlichen Maß «, der Schaffung öffentlicher Räume zur sozialen Interaktion und sozialen Kontrolle und somit zum »community building «. Die dafür notwendige geringere Ausnutzung der Fläche für Wohnraum wird bewusst in Kauf genommen. Nach den Vorstellungen des für HOPE VI ursprünglich verantwortlichen Behördenleiters von HUD sollte dennoch allen Bewohnern eine bezahlbare Unterkunft vermittelt werden, sei es in den neuen Einheiten, sei es über Berechtigungsscheine auf dem privaten Wohnungsmarkt. ${ }^{37}$

Dass HOPE VI in der Tat eine für die USA grundlegende Neuausrichtung der Idee von » Public Housing « verfolgt, wird auch daran deutlich, dass eigens einige Gesetze geändert werden mussten, die die anvisierte niedrigere Bebauungsdichte und die Akzeptanz von Berechtigungsscheinen behinderten. Abgeschafft wurde die »onefor-one rule", die für jede abgerissene Einheit von »Public Housing « eine neu errichtete gefordert hatte. Aufgehoben wurde ebenfalls die »take one, take all «Regel, die Hauseigentümer, die einen Mieter mit Berechtigungsschein akzeptierten, dazu verpflichtete, auch andere Bewerber mit »vouchers " aufzunehmen (was faktisch jedoch die Bereitschaft dazu eher dämpfte).

Laut Thomas Kingsley vom Urban Institute gilt HOPE VI vielen Beobachtern als »die beeindruckendste Initiative von HUD zur Quartiererneuerung «. Insgesamt 8,5 Milliarden Dollar wurden und werden in 240 Nachbarschaften investiert, um »111,059 neue und erneuerte Wohneinheiten bereitzustellen, davon 59,674 für Familien mit niedrigem Einkommen und Anrecht auf eine Sozialwohnung «.38

MTO wiederum stellt ein Pilotprojekt dar, das gezielt die sozialen Folgen der Umsiedlung von Familien aus Gebieten mit hoher Armutskonzentration in Gebiete mit niedriger Armutskonzentration prüfen sollte. Drei Vergleichsgruppen wurden aus insgesamt 4.610 Familien mit sehr niedrigem Einkommen gebildet, die in fünf

35 Ich übernehme hier wie an anderer Stelle den amerikanischen Begriff, weil sich, wie bereits angesprochen, Konzept und Realität von »Public Housing « zumindest in der Vergangenheit deutlich vom öffentlichen Wohnungsbau in Deutschland unterschieden.

36 Zur Geschichte des Programms siehe Cisneros 2009; Katz 2009.

37 Vgl. Cisneros 2009, S. 8.

38 Kingsley 2009 a, S. 263. 
Metropolengebieten ${ }^{39}$ in »Public Housing « wohnten: MTO-Gruppe mit Berechtigungsscheinen, der Verpflichtung, in Gebiete mit niedriger Armutsquote (Armutsanteile unter 10 Prozent) zu ziehen, und Hilfestellungen (Beratung der Umsiedler sowie Einwerbung potentieller Vermieter); eine Vergleichsgruppe mit Berechtigungsscheinen, aber ohne Auflage bezüglich des Umzugsgebiets und ohne Hilfestellungen; eine Kontrollgruppe im Armutsgebiet verbleibender Mieter.

\section{Soziale Auswirkungen der Programme}

Beide Programme zeitigten einige deutlich positive Effekte. Infolge von HOPE VI und vergleichbaren Initiativen kam es zu einem Rückgang der Konzentration von »Public Housing « in Gebieten mit hoher Armutsdichte. Die meisten der großen Projekte wurden abgerissen, die Wohnungsstandards in »Public Housing " verbessert. ${ }^{40}$ Die Nutzer von Berechtigungsscheinen zogen meist langfristig, auch über Wohnungswechsel hinweg, in Gebiete mit niedrigerer (wenn auch selten niedriger) Armutsrate. Bei ihnen nahm die Zufriedenheit mit den Wohnbedingungen allgemein zu. Crowley geht allerdings davon aus, dass nur wenig mehr als die Hälfte aller durch die Umsiedlungsmaßnahmen von HOPE VI betroffenen Haushalte in dieser Weise profitierten. ${ }^{41}$

Beide Programme weisen aber auch deutliche Schwächen auf. Bei HOPE VI sind diese Schwächen darüber hinaus mit sehr problematischen Folgen verbunden. Sie betreffen zunächst das Angebot von erschwinglichem Wohnraum. Der Rückgang der Einheiten von »Public Housing « nach Abriss und nur unvollständigem Ersatz verengt das Wohnungsangebot für Bezieher niedriger Einkommen weiter. Seit 1994 ging die Zahl der Wohnungen in »Public Housing « stetig zurück, bis 2008 verschwanden rund 270.000 Einheiten. ${ }^{42}$ Nur 55 Prozent der unter HOPE VI abgerissenen oder rehabilitierten Wohnungen werden durch neue Einheiten von »Public Housing " ersetzt, allerdings 81 Prozent der zuvor bewohnten Einheiten. ${ }^{43}$ Lediglich ein Viertel der umgesiedelten Haushalte kehrte bis September 2008 in ihre durch HOPE VI umstrukturierte Nachbarschaft zurück, wenig mehr werden noch erwartet. ${ }^{44}$ Kingsley schätzt, dass in den USA weniger als 30 Prozent der Bezieher niedriger Einkommen, die eigentlich einer öffentlichen Unterstützung bei der Wohnungsversorgung bedürften (einschließlich, aber nicht nur durch »Public Housing «), solche 2005 auch erhielten. ${ }^{45}$

Die Nutzungsquote (Realisierung) von Berechtigungsscheinen wiederum hängt vom privaten Angebot an erschwinglichem Wohnraum ab und damit von den kon-

39 Es handelt sich um Baltimore, Boston, Chicago, Los Angeles und New York.

40 Vgl. Schwartz 2010, S. 151 f.; Kingsley 2009 a, S. 264.

41 Crowley 2009, S. 232.

42 Vgl. Schwartz 2010, S. 126.

43 Ebd., S. 145.

44 Vgl. Kingsley 2009 b, S. 302.

45 Kingsley 2009 a, S. 269. 
junkturellen Spielräumen und Diskriminierungen am Wohnungsmarkt - beides schränkt den angestrebten Erfolg von sozialräumlicher Mischung durch HOPE VI bereits von vornherein weiter ein. Viele ehemalige Bewohner der Projekte, die nun mit Berechtigungsscheinen ausgestattet wurden, hatten erhebliche Probleme, mit diesen umzugehen und sie erfolgreich einzusetzen. Häufig fehlte es an der notwendigen Beratung und Unterstützung. ${ }^{46}$

Zudem werden bestimmte ehemalige Bewohnergruppen durch verschärfte soziale Zulassungskriterien (zum Beispiel keine Kriminalität in der Familie, geprüfte Fähigkeit, einen Haushalt zu führen, keine Kreditprobleme; in Chicago: Erwerbstätigkeit von mindestens 30 Wochenstunden oder Vollzeitteilnahme an Schule bzw. Ausbildung) von den neu gebauten oder erneuerten Wohneinheiten ausgeschlossen und verlieren damit ihre Wohnung. ${ }^{47}$ Überdies wächst der Aufwertungs- und Privatisierungsdruck auf die Gebiete mit erneuertem »Public Housing «-Bestand und einer Zunahme von Haushalten mit mittlerem Einkommen. ${ }^{48}$ Wie eine Fallstudie aus Chicago zeigt, verstärkt dies die Befürchtung unter den betroffenen Bewohnern, aus ihrer Nachbarschaft verdrängt zu werden. Diese Befürchtung ist umso realistischer, je länger sich der Prozess der Umgestaltung hinzieht, Bewohner deshalb zwischenzeitlich anderweitig untergebracht werden müssen und eine Rückkehr bereits dadurch unwahrscheinlicher wird. ${ }^{49}$ Insgesamt mussten zwischen 1993 und 2007 über 72.000 Haushalte infolge von HOPE VI umsiedeln. ${ }^{50}$

Erhebliche Kritik von Bewohnern macht sich deshalb an den Entscheidungsverfahren (beispielsweise bei der Auswahl der abzureißenden Wohnanlagen), der zu geringen Unterstützung bei der Wohnungssuche und der Vernachlässigung der Infrastruktur in den Gebäuden und in der Nachbarschaft, solange sie vor allem von armen Familien genutzt wurde, fest.

Weder HOPE VI noch MTO haben schließlich die ethnische/rassische Segregation überwinden können. Die Mehrheit der im Rahmen dieser Programme Umgesiedelten leben nach wie vor in Nachbarschaften mit überwiegender »Minority«-Bevölkerung. ${ }^{51}$ Wie weit dies auf Diskriminierung am Wohnungsmarkt oder eigene Wahl zurückgeht, bliebe zu klären.

Ein zweiter, großer Problembereich liegt im begrenzten Fokus auf Nachbarschaftseffekte (soziale Veränderungen allein durch Umsiedlung), ohne direkte Hilfen zur Überwindung der Armut. Dadurch bleiben Probleme der nicht direkt adressierten individuellen Armut auch im neuen Wohnumfeld bestehen. Das zeigen etwa die Schwierigkeiten vieler Bezieher von Berechtigungsscheinen, die zuvor im »Public Housing « inbegriffenen Nebenkosten zu begleichen und sich ausreichend Lebens-

46 Vgl. Cisneros 2009, S. 9; Levy, Gallagher 2006.

47 Vgl. Schwartz 2010, S. 147.

48 Vgl. Cisneros 2009, S. 13.

49 Vgl. Levy, Gallagher 2006.

50 Vgl. Kingsley 2009 b, S. 302.

51 Vgl. Popkin, Cunningham 2009, S. 196; Schwartz 2010, S. 148, 202.

Leviathan, 40. Jg., 3/2012 
mittel zu kaufen. ${ }^{52}$ Soziale Verbesserungen bezüglich Ausbildung, Einkommen und Beschäftigung sind nicht direkt Gegenstand von HOPE VI, allerdings werden sie implizit angestrebt und erwartet. Eine weitere in Chicago durchgeführte Studie zeigt hingegen kaum Verbesserungen in der Beschäftigungs- und Armutssituation von Bewohnern von »Public Housing « nach der Implementation von HOPE VI. Vor allem Gesundheitsprobleme und geringe Qualifikation stehen, von der »Angebotsseite " der Individuen her betrachtet, der Beschäftigung im Weg. ${ }^{53}$ Auch für die Umsiedler im Rahmen von HOPE VI änderte sich die Ausgangslage nicht. ${ }^{54}$

Ausdrücklich und differenziert werden die sozialen Folgen von Umsiedlungsmaßnahmen im Programm MTO getestet, mit zwiespältigen Ergebnissen. Im Kern bestätigen sie allerdings die Befunde von HOPE VI. Die positiven Effekte in den Bereichen Wohnzufriedenheit und Sicherheit werden nicht von ähnlich positiven Auswirkungen in den Bereichen (Aus-)Bildung, Arbeitsstellen und Einkommen begleitet. Evaluationen, die im Wesentlichen auf Vergleichen zwischen der MTO-Gruppe (1.820 Haushalte) und der in Vierteln mit hoher Armutskonzentration verbleibenden Kontrollgruppe (1.440 Haushalte) beruhen, zeitigen die folgenden zentralen Ergebnisse:

Mobilität: Fünf Jahre nach dem Wegzug aus einem Gebiet mit extrem hoher Armutsdichte lebte die Mehrheit der Umsiedler wieder in einer Nachbarschaft mit hoher Armut, wenn auch nicht mehr in Gebieten mit einer extrem hohen Armut. Das Ziel, dauerhaft in eine Nachbarschaft mit geringer Armutsquote umzuziehen und sich dort zu integrieren, wurde nur von wenigen MTO-Haushalten erreicht.

Messbare Effekte bei der Verbesserung der Arbeitsmarktsituation konnten nicht festgestellt werden. Die Umzüge führten nicht notwendigerweise in die Nähe von Gebieten mit einem großen Angebot von Arbeitsplätzen für gering qualifizierte Neueinsteiger. Auch beim Einkommen waren insgesamt keine Verbesserungen erkennbar.

Sicherheit: Übereinstimmend werden eine höhere wahrgenommene und reale Sicherheit sowie ein höheres Maß an sozialer Ordnung innerhalb der neuen Nachbarschaften mit geringer Armutsrate festgestellt. ${ }^{55}$ Der außerdem verzeichnete Anstieg des allgemeinen Wohlbefindens und der Zufriedenheit der MTO-Teilnehmer in der Versuchsgruppe ist zu großen Teilen mit den positiven Erfahrungen bei der wichtigsten Motivation der Bewerber für die Teilnahme am MTO-Experiment zu erklären: dem Wunsch nach erhöhter Sicherheit im eigenen Wohnumfeld. Der Umzug trug dazu bei, die Lebensqualität von armen Familien zu steigern, indem sie vor der Gewalt in den Ghetto-Nachbarschaften geschützt werden. Dies ist wirklich ein positiver Effekt des anderen Ortes, auch ohne Einbindung in neue Netzwerke.

52 Vgl. Schwartz 2010, S. 148; Popkin, Cunningham 2009, S. 198 f. Auf ähnliche Probleme weist Levy auch für nicht umgesiedelte Bewohner einer renovierten Wohnanlage in Chicago hin (Levy 2010).

53 Vgl. Levy 2010.

54 Vgl. Popkin, Cunningham 2009, S. 197.

55 Vgl. Rosenbaum et al. 2003; Ludwig et al. 2008; Galster 2003; Briggs et al. 2010. 
Frauen und Mädchen: Übereinstimmend positiv werden in den Evaluationen auch die Veränderungen bewertet, die durch die Umzüge im Rahmen von MTO für Mädchen und junge Frauen erzielt wurden. ${ }^{56}$ Die erlebte Sicherheit, das dadurch verbesserte physische und psychische Wohlergehen sowie das Verhalten der Mädchen der Versuchsgruppe weisen deutliche Unterschiede im Vergleich zu den in sehr armen Gebieten verbliebenen Bewohnern auf. Insgesamt haben die Mädchen von der Veränderung der Wohnumgebung deutlich mehr profitiert als die männlichen Jugendlichen. Bei jungen Frauen war ein Absinken von Delinquenz zu beobachten, während das bei jungen Männern nicht der Fall war. Bei ihnen hat sich die Delinquenzrate nach dem Umzug sogar vergrößert.

Die negativen Veränderungen bei männlichen Jugendlichen zeigen sich für die Versuchsgruppe im Vergleich zur Kontrollgruppe an der höheren Anzahl von Verhaftungen wegen Eigentumsdelikten. Sampson erwähnt darüber hinaus einen negativen Effekt auf die physische Gesundheit von jungen Männern der Versuchsgruppe. ${ }^{57}$

Psychisches Wohlbefinden: Einhellig werden als positive, MTO-bedingte Effekte Verbesserungen des psychischen Wohlbefindens von Erwachsenen innerhalb der Versuchsgruppe konstatiert. Unterschiede bestehen allerdings hinsichtlich des Stellenwerts, der diesen Verbesserungen beigemessen wird. Clampet-Lundquist und Massey bewerten die Erfolge aufgrund der von ihnen diagnostizierten Schwäche des MTO-Programms hinsichtlich langfristiger Veränderungen und fehlender sozioökonomischer Verbesserungen als eher unbedeutend. Ludwig et al. und Sampson hingegen erwarten anhaltend positive Folgewirkungen. ${ }^{58}$

Veränderung des Kontextes: Grundlage für MTO waren sehr optimistische Annahmen darüber, was die Nachbarschaften mit höheren Einkommen in Bezug auf Rollenmodelle, auf die Vermittlung von Arbeitsstellen und die Stiftung starker Gemeinschaften (»communities«) leisten würden. Die Erwachsenen sollten einen leichteren Zugang zu Arbeitsplätzen außerhalb der Innenstadt finden und wertvolle Ressourcen aus neuen sozialen Beziehungen schöpfen können. Aber viele der Umsiedler hielten an ihren bestehenden Netzwerken zu männlichen Verwandten in den Herkunftsquartieren fest, von denen ein erheblicher Anteil ehemals straffällig oder noch aktuell in kriminelle Aktivitäten involviert war.

Soziale Netze: Die MTO-Familien sollten nach dem Umzug neue und für sie nützliche Bindungen in den »besseren « Quartieren entwickeln. Dies gelang jedoch nur selten. Den meisten MTO-Teilnehmern blieben die sozialen Welten in den neuen Nachbarschaften unzugänglich, ihre eigene soziale Welt war weiter in ihre Herkunftsgebiete eingebettet. Ein aktives soziales Leben führten sie mit ihren alten Freunden. Nur wenige nutzten den Umzug, um Distanz und Abstand zwischen sich und den Zumutungen und Risiken der sozialen Bezüge in den »Projects « zu bringen. Die anhaltende soziale Segregation der Netzwerke trotz der größeren Heterogenität

56 Vgl. Popkin et al. 2008.

57 Vgl. Shroder 2003; Sampson 2008.

58 Clampet-Lundquist, Massey 2008; Ludwig et al. 2008; Sampson 2008.

Leviathan, 40. Jg., 3/2012 
der neuen Nachbarschaften wird von den Evaluatoren als eine der stärksten Enttäuschungen des Experiments bewertet. ${ }^{59}$ Der räumliche Kontext wurde verändert, aber der soziale Kontext oft aufrechterhalten. So veränderte sich zwar der Wohnort, aber nicht die "community «.

Zusammenfassend muss man feststellen, dass die Nachbarschaften sich verbessert haben, aber die neuen Nachbarn hatten kaum einen positiven Einfluss auf die soziale Situation der umgezogenen Haushalte. Die Abwesenheit von alltäglicher Gewalt und Kriminalität wurde zwar als wichtiger und wesentlicher Erfolg bewertet, aber die sozialen, benachteiligenden Kontexteffekte wurden nicht neutralisiert.

Dass MTO fortgesetzt oder gar ausgeweitet wird, erscheint zweifelhaft. Zu stark scheinen die politischen Widerstände dagegen zu sein, in Zeiten weiterer Kürzungen im Sozialbereich die bei MTO anfallenden zusätzlichen Kosten für Beratung zu finanzieren - obgleich gerade die Hilfestellungen zu deutlich besseren Erfolgen bei der Wohnungssuche geführt hatten als die Vergabe von Berechtigungsscheinen ohne weitere Unterstützung. Aber auch soziale Widerstände in Vierteln mit niedriger Armutsquote, arme Familien aufzunehmen, haben bereits beim bisherigen Programm eine Ausweitung verhindert. ${ }^{60}$

\section{Lehren aus der amerikanischen Politik zur Dekonzentration der Armut}

Welche Lehren lassen sich aus HOPE VI und MTO ziehen, die auch für die deutsche und europäische Diskussion von Bedeutung sind?

Die erste betrifft das Angebot von erschwinglichem Wohnraum und den Grundwiderspruch von HOPE VI und MTO. Dieser besteht darin, dass einerseits die Dekonzentration von Armut angestrebt wurde, dies aber andererseits unter der Rahmenbedingung stattfinden sollte, »Public Housing " abzuschaffen bzw. den Umfang des öffentlichen Eigentums an Wohnungen zu reduzieren und die Wohnungsversorgung von bedürftigen Haushalten über den privaten Markt zu organisieren. Das sind nach den Erfahrungen mit HOPE VI und MTO zwei Ziele, die sich nicht vertragen: Entweder schafft man den öffentlichen Wohnungsbestand ab und nimmt dann eine räumliche Konzentration von Armen in bestimmten (unattraktiven) Nachbarschaften in Kauf; oder man will die Kontexteffekte vermeiden, die mit der Konzentration von Armut verbunden sind, aber dann muss man dauerhafte, bezahlbare Wohnmöglichkeiten für sie in solchen Quartieren schaffen, die den Bewohnern bessere Lebenschancen bieten können. Die Verringerung der Einheiten von »Public Housing « durch HOPE VI bei gleichzeitiger Erhöhung des Anteils von Wohnungen für höhere Einkommen verstärkt die Gefahr der Verdrängung durch Aufwertung der HOPE VI-Gebiete für die Bezieher niedriger Einkommen. Da zudem die Nutzer von Berechtigungsscheinen in ihrer Mobilität auf die Verfügbarkeit erschwinglichen Wohnraums auf den privaten Wohnungsmärkten angewiesen sind, geht in Zeiten und Regionen mit »engen « Wohnungsmärkten das Angebot im

59 Vgl. Cove et al. 2008; Ferryman et al. 2008; Galster 2003; Briggs et al. 2010.

60 Vgl. Schwartz 2010, S. $202 \mathrm{f}$. 
unteren Mietpreissegment weiter zurück. Beides verschärft die Wohnungsproblematik für Menschen mit niedrigem Einkommen. ${ }^{61}$ Nur ein sozialer Wohnungsbau in allen Bereichen der Stadt, das heißt preiswerte und diskriminierungsfrei vergebene Wohnungen, können für faire Chancen und eine größere Wahlfreiheit auch derjenigen Haushalte sorgen, die es sich aufgrund ihrer sozialen Lage nicht aus eigener Kraft leisten können, in attraktiven Quartieren eine Wohnung zu mieten oder zu kaufen. Diese Lehre muss auch in Deutschland und anderen europäischen Ländern wieder gelernt werden, in denen der öffentliche Wohnungsbestand kontinuierlich reduziert wurde und wird.

Die zweite Lehre ergibt sich aus den Grenzen einer Strategie, die Armut allein von den Quartierseffekten her überwinden will. Was die sozialen Folgen von HOPE VI und MTO betrifft, so zeigt sich ein uneinheitliches und widersprüchliches Bild. Für Umsiedler in Viertel mit niedrigerer oder gar niedriger Armutsquote verbessern sich in der Regel die Wohnbedingungen und insbesondere das Gefühl der Sicherheit für sich selbst und die Familienangehörigen. Das bestätigen sowohl Evaluationen von HOPE VI als auch von MTO. ${ }^{62}$ Dekonzentration von Armut ist bereits deshalb ein erstrebenswertes Ziel.

Die weit weniger befriedigenden Resultate hinsichtlich Beschäftigung, Einkommen und materieller Bewältigung des Alltags resultieren allerdings zum Teil als unbeabsichtigte Nebenfolgen gerade aus der Umsiedlung selbst - sei es, weil sich in erreichbarer Nähe von Wohngebieten mit niedriger Armutsrate auch weniger Arbeitsplätze für gering Qualifizierte finden, sei es, weil die Vereinbarkeit von Berufstätigkeit und Kinderbetreuung oder Pflegeverpflichtungen in der Familie wegen längerer und teurerer Verkehrswege oder auch fehlender Unterstützung aus den früheren familiären sozialen Netzen schwieriger wird. Der Ausgleich zwischen unterschiedlichen, lebensnotwendigen Anforderungen im Alltag stellt gerade für Menschen und Haushalte mit geringem Einkommen ein besonderes zeitliches und finanzielles Problem dar, das durch die Umsiedlung verschärft werden kann. ${ }^{63}$ Letzteres gilt insbesondere für die Gruppe der Umsiedler, die weiterhin an ihrem alten Arbeitsplatz beschäftigt sind.

Überdies wird die Erwartung, dass sich in neuen Nachbarschaften auch neue soziale Kontakte ergeben, die Aufstiegsmöglichkeiten eröffnen, durch die sozialen Grenzen durchkreuzt, die die ärmeren Neuankömmlinge von den bessergestellten Etablierten trennen. Dieser Befund bestätigt Erfahrungen aus Quartieren in amerikanischen Städten, in denen umgekehrt wohlhabende Zugezogene zumindest für eine Weile neben armen »Ortsansässigen « leben, ohne dass dies zu einer Verbindung der sozialen Verkehrskreise führen würde, ${ }^{64}$ aber auch aus sozial gemischten europäischen Vierteln mit hohen Armutsanteilen. ${ }^{65}$

61 Vgl. Comey et al. 2008; Crowley 2009, S. 237-240; Schwartz 2010, S. 207.

62 Vgl. Schwartz 2010, S. 148, 200; Cahill et al. 2011.

63 Vgl. Cove et al. 2008; Comey et al. 2008.

64 Vgl. Freeman 2006, S. 164, 204.

65 Vgl. Burgers 2009, S. 136 f.; Neef, Keim 2007, S. 289.

Leviathan, 40. Jg., 3/2012 
Somit erfüllen weder HOPE VI noch MTO die Hoffnung, allein durch Ortsveränderung und eine stärkere soziale Mischung im Quartier Haushalte aus der Armut herauszubringen und zu stabilisieren. Sie war von vornherein unangemessen, weil sie einem zu partiellen, verengten Blick auf Nachbarschaft entspringt. Er blendet nicht nur die Folgen sozialer Ungleichheiten in der neuen Wohnumgebung aus, sondern auch das komplexe Zusammenwirken von Arbeitsmärkten, familiären Verpflichtungen, Verkehrswegen, sozialstaatlichen Dienstleistungen und sozialen Netzen - kurz: die »institutionellen Komplementaritäten "66, die innerhalb und außerhalb von Quartieren soziale Chancen eröffnen oder verschließen.

Um Armut überwinden zu können, müssen nicht nur entsprechende Arbeitsplätze angeboten werden (siehe Kapitel 3); diese müssen auch verkehrstechnisch zu erschwinglichen Preisen erreichbar sein. Familiäre und berufliche Verpflichtungen müssen für Haushalte mit knappen finanziellen Mitteln in Einklang zu bringen sein; das erfordert soziale und institutionelle Entlastungen. Damit Kontakte über Klassen- und Schichtungsgrenzen geknüpft und zu einer verlässlichen, weil dauerhaften Ressource werden können, bedarf es besonderer, organisierter Anlässe und institutionalisierter Anreize, etwa in Schulen und Vereinen. Kinder und Jugendliche aus armen Familien werden nicht allein dadurch bereits lernfähiger, dass sie in eine Schule mit Kindern aus höheren sozialen Klassen versetzt werden. Sie müssen sich dort auch einleben können, die Schule muss auch institutionell darauf abzielen, die immer wieder durch die Pädagogik erwiesenen Potentiale gemeinsamen Lernens zu nutzen, und die Eltern müssen bereit sein, dies mitzutragen. Kurz: Nachbarschaften müssen erst zu Nachbarschaften der Möglichkeiten (»opportunities «) werden. Diese Möglichkeiten zu definieren hatte MTO jedoch versäumt, zumindest aber im Ungefähren gelassen.

Gerade weil HOPE VI und MTO so ausschließlich auf soziale Mischung setzen, um städtische Armut zu überwinden, sind sie so lehrreich auch für die europäische Diskussion. Sie zeigen geradezu experimentell die Grenzen dieses Ansatzes auf. Weder die Förderung der Mobilität von armen Haushalten noch Bestrebungen, die Mittelklassen wieder in verarmten Innenstadtgebieten anzusiedeln, ${ }^{67}$ reichen für sich aus, um für arme Haushalte positive Nachbarschaftseffekte zu erzeugen.

Unzulässig wäre aber auch der Umkehrschluss - dass die räumliche Konzentration von Armut unerheblich sei. Ebenso wenig lässt sich schlussfolgern, das MTO-Experiment sei gescheitert. Gescheitert sind die unrealistischen Erwartungen einer allein auf die räumliche Mobilität gerichteten Strategie. Denn diese hat einerseits nur den Erstumzug unterstützt und die Re-Konzentration der armen Haushalte (wenn auch in Nachbarschaften mit einem durchschnittlich niedrigeren Armutsniveau als die Herkunftsquartiere) dann nicht verhindern können; sie hat andererseits die Bedeutung und Beharrlichkeit sozialer Bindungen unterschätzt, die umso mehr zunahmen, je schwieriger sich die Integration der MTO-Teilnehmer an ihren neuen Wohnorten gestaltete; sie hat schließlich drittens die institutionellen Ressourcen innerhalb und außerhalb von Nachbarschaften vernachlässigt, die notwendig sind und ineinan-

66 Hall, Soskice 2001, S. 17.

67 Zum Beispiel Rotterdam: Burgers 2009; Snel, Engbersen 2009. 
dergreifen müssen, um Armut zu überwinden. Mobilitätsförderung kann eine soziale Wohnungspolitik und Sozialpolitik nicht ersetzen.

Deshalb sprechen die amerikanischen Erfahrungen für die Notwendigkeit einer Verbindung von Maßnahmen zur Dekonzentration von Armut mit infrastrukturellen und individuellen Maßnahmen zur Unterstützung der armen Haushalte in ihren Strategien, aus der Armut herauszukommen, sowie mit nachbarschaftsbezogenen Maßnahmen, die Vernetzungen über soziale Grenzen hinweg erst ermöglichen. Dies gilt unabhängig davon, wie eine stärkere soziale Mischung im Quartier zustande kommt - ob durch Umsiedlung ärmerer Familien in wohlhabendere Viertel oder umgekehrt durch die Zuwanderung wohlhabenderer Familien in eine arme Nachbarschaft. Die Anerkennung der Vielschichtigkeit der Aufgaben macht politische Intervention nicht einfacher - aber vielleicht realitätstüchtiger und zielstrebiger.

Das Bund-Länder-Programm »Soziale Stadt « stellt seit 1999 in Deutschland einen ersten, wichtigen Schritt in Richtung auf eine quartiersbezogene Politik dar, die jener Vielschichtigkeit der Problemlagen Rechnung zu tragen versuchte. Allerdings richtete sich das Programm ausschließlich auf die Verbesserung von Lebensbedingungen in den Quartieren selbst und nicht auf die Mobilität ihrer Bewohner. Sein Anspruch, ressortübergreifend Kräfte der lokalen Verwaltung und Politik zu bündeln, war angemessen und hätte noch stärker die Bereiche Arbeitsmarkt-, Ausbildungs- und Schulpolitik einbeziehen können und müssen. Statt es dafür entsprechend mit konsumtiven Mitteln weiter auszustatten, wurde das Programm indessen trotz erkennbarer Erfolge auf ein Minimum rein investiver Mittel eingekürzt und damit insgesamt in Frage gestellt. ${ }^{68}$

Die dritte Lehre aus HOPE VI und MTO besteht einmal mehr in der Anerkennung der großen Bedeutung, die der Einbeziehung der Bewohner in die Entscheidungsprozesse und bei deren Umsetzung zukommt. Mangelnde Transparenz, unangemessene Entscheidungen darüber, welche Wohnanlagen abzureißen sind, zu geringe Unterstützung bei der Umsiedlung und der Auseinandersetzung mit deren Folgen wurden zwar im Verlauf der Implementation von HOPE VI als Probleme erkannt, jedoch nicht oder allenfalls graduell behoben. Partizipation aber ist entscheidend für jedes politische Projekt, das auf die Stärkung der Handlungsmöglichkeiten von Menschen abzielt, somit gerade auch für arme Haushalte.

Besonders kritisch zu bewerten ist HOPE VI schließlich dort, wo es gezielt sozial ausgrenzend wirkt. Die verschärften Zugangskriterien zu »Public Housing «, einschließlich einer Sippenhaftung für Familienangehörige mit Vorstrafen, und die Anwendung der Unterscheidung zwischen würdigen (arbeitenden) und nicht würdigen (arbeitslosen) Armen auf die Verteilung bzw. Verweigerung von Wohnraum (Fall Chicago) passen sich ein in die Logik der »welfare reform « von 1996, die noch schärfer als zuvor eine Linie zwischen unterstützungsberechtigten und zu bestrafenden Armen zieht. Sie bedrohen Hilfsbedürftige mit Ausgrenzung in ihrer radikalsten Form, durch Rechtlosigkeit, in einem zentralen Bereich menschlicher Bedürfnisse, dem Wohnen. Ohne sozialstaatliche Anerkennung und tatsächliche Garantie eines Rechts auf kulturell angemessenen Wohnraum für jedermann, auch 
in den kritischen Phasen einer provisorischen Unterbringung, wird jede Politik der Dekonzentration von Armut, die Umsiedlungen in Kauf nimmt, die am meisten Bedürftigen noch weiter schädigen.

Eine grundsätzliche Kritik an HOPE VI besteht darin, dass das Programm eher den Nutznießern steigender Immobilienpreise in den betroffenen Quartieren zugute kommt als deren Bewohnern. ${ }^{69}$ Befürworter des Programms wiederum betonen die Chancen für eine Verbesserung der Lebensbedingungen, räumen aber ein, dass es dafür noch erheblicher Änderungen in den Rahmenbedingungen und der Implementation bedarf. Beides ist im Spiel. Die Faktoren aber, von denen es abhängt, ob eine Politik der Dekonzentration von Armut stärker in die eine oder andere Richtung wirkt, lassen sich nach den amerikanischen Erfahrungen sehr viel genauer benennen. Gerade deshalb sind sie für die europäische Diskussion so wertvoll.

\section{Literatur}

Berube, Alan; Frey, William H. 2005. »A decade of mixed blessings. Urban and suburban poverty in Census 2000 ", in Redefining urban and suburban America. Evidence from Census 2000. Volume Two, hrsg. v. Berube, Alan; Katz, Bruce; Lang, Robert E., S. 111-136. Washington D.C.: The Brookings Institution Press.

Briggs, Xavier de Souza 2005. "More pluribus, less unum? The changing geography of race and opportunity «, in The geography of opportunity. Race and housing choice in metropolitan America, hrsg. v. Briggs, Xavier de Souza, S. 17-41. Washington D.C.: The Brookings Institution Press.

Briggs, Xavier de Souza; Popkin, Susan J.; Goering, John M. 2010. Moving to opportunity. The story of an American experiment to fight ghetto poverty. New York: Oxford University Press.

Burgers, Jack 2009. "Changing societies by rebuilding neighbourhoods: on the effects of restructuring deprived urban areas - experiences from the Netherlands", in Between the social and the spatial. Exploring the multiple dimensions of poverty and social exclusion, hrsg. v. De Boyer, Katrien; Dewilde, Caroline; Dierckx, Danielle; Friedrichs, Jürgen, S. 133-148. Farnham, Burlington: Ashgate.

Cahill, Meagan; Lowry, Samantha; Downey, P. Mitchell 2011. Movin' out. Crime displacement and HUD's HOPE VI initiative. The Urban Institute, Research Report August 2011. Washington D.C.: The Urban Institute.

Cisneros, Henry G. 2009. » A new moment for people and cities «, in From despair to hope: HOPE VI and the new promise of public housing in American cities, hrsg. v. Cisneros, Henry G.; Engdahl, Lora, S. 3-13. Washington D.C.: The Brookings Institution Press.

Clampet-Lundquist, Susan; Massey, Douglas S. 2008. "Neighborhood effects on economic selfsufficiency: a reconsideration of the moving to opportunity experiment ", in American Journal of Sociology 114, S. 107-143.

Comey, Jennifer; Briggs, Xavier de Souza; Weismann, Gretchen 2008. Struggling to stay out of high-poverty neighborhoods: lessons from the moving to opportunity experiment. The Urban Institute, Metropolitan Housing and Communities Center, Brief No. 6, March 2008. Washington D.C.: The Urban Institute.

Coulton, Claudia; Theodos, Brett; Turner, Margery A. 2009. Familiy mobility and neighborhood change. New evidence and implications for community initiatives. The Urban Institute, Making Connections Research Series. Washington D.C.: The Urban Institute.

Cove, Elizabeth; Turner, Margery A.; Briggs, Xavier de Souza; Duarte, Cynthia 2008. Can escaping from poor neighborhoods increase employment and earnings? The Urban Institute, Metropolitan Housing and Communities Center, Brief No. 4, March 2008. Washington D.C.: The Urban Institute.

69 Vgl. Crowley 2009, S. 230. 
Crowley, Sheila 2009. "HOPE VI: what went wrong? «, in From despair to hope: HOPE VI and the new promise of public housing in American cities, hrsg. v. Cisneros, Henry G.; Engdahl, Lora, S. 229-247. Washington D.C.: The Brookings Institution Press.

DeNavas-Walt, Carmen; Proctor, Bernadette D.; Smith, Jessica C. 2011. US Census Bureau, current population reports, P 60-239, Income, poverty and health insurance coverage 2010. Washington D.C.: US Government Printing Office.

Dreier, Peter; Mollenkopf, John; Swanstrom, Todd 2004. Place matters. Metropolitics for the twenty-first century. Revised second edition. Lawrence: University Press of Kansas.

Fasenfest, David; Booza, Jason; Metzger, Kurt 2006. »Living together: a look at racial and ethnic integration in metropolitan neighborhoods, 1990-2000 ", in Redefining urban and suburban America. Evidence from Census 2000. Volume Three, hrsg. v. Berube, Alan; Katz, Bruce; Lang, Robert E., S. 93-117. Washington D.C.: The Brookings Institution Press.

Ferryman, Kadija S.; Briggs, Xavier de Souza; Popkin, Susan J.; Rendón, María 2008. Do better neighborhoods for MTO families mean better schools? Three-city study of moving to opportunity. The Urban Institute, Brief No. 3. Washington D.C.: The Urban Institute.

Fortuny, Karina; Chaudry, Ajay; Jargowsky, Paul 2010. Immigration trends in metropolitan America, 1980-2007. The Urban Institute, Brief No. 1. Washington D.C.: The Urban Institute.

Freeman, Lance 2006. There goes the hood. Views of gentrification from the ground up. Philadelphia: Temple University Press.

Frey, William F. 2005. »The new great migration. Black Americans' return to the South, 1965-2000", in Redefining urban and suburban America. Evidence from Census 2000. Volume Two, hrsg. v. Berube, Alan; Katz, Bruce; Lang, Robert E., S. 87-110. Washington D.C.: The Brookings Institution Press.

Galster, George 2003. "The effects of MTO on sending and receiving neighborhoods ", in Choosing a better life? A social experiment in leaving poverty behind, hrsg. v. Goering, John; Richardson, Todd M.; Feins, Judith D., S. 365-382. Washington D.C.: Urban Institute Press.

Goering, John 2005. »Expanding housing choice and integrating neighborhoods: the MTO experiment ", in The geography of opportunity. Race and housing choice in metropolitan America, hrsg. v. Briggs, Xavier de Souza, S. 127-149. Washington D.C.: Brookings Institution Press.

Häußermann, Hartmut 2012. »Die Zeit von Bibliotheken für Mädchen mit Migrationshintergrund ist vorbei. Zu den Folgen der Mittelkürzungen im Programm Soziale Stadt ", in Forum Stadt 39, 1, S. 90-96.

Häußermann, Hartmut; Kronauer, Martin 2009. »Räumliche Segregation und innerstädtisches Ghetto", in Inklusion und Exklusion: Analysen zur Sozialstruktur und sozialen Ungleichheit, hrsg. v. Stichweh, Rudolf; Windolf, Paul, S. 157-173. Wiesbaden: VS Verlag für Sozialwissenschaften.

Hall, Peter A.; Soskice, David 2001. "Introduction to varieties of capitalism ", in Varieties of capitalism. The institutional foundations of comparative advantage, hrsg. v. Hall, Peter A.; Soskice, David, S. 1-70. Oxford, New York: Oxford University Press.

Iceland, John 2006. Poverty in America. A handbook. Second edition. Berkeley, Los Angeles, London: University of California Press.

Jargowsky, Paul A. 2003. Stunning progress, hidden problems: the dramatic decline of concentrated poverty in the $1990 \mathrm{~s}$. The Brookings Institution, Center on Urban and Metropolitan Policy, The Living Cities Census Series, May 2003. Washington D.C.: The Brookings Institution.

Jargowsky, Paul A.; Yang, Rebecca 2006. »The >underclass« revisited: a social problem in decline ", in Journal of Urban Affairs 28, 1, S. 55-70.

Katz, Bruce 2009. "The origins of HOPE VI«, in From despair to hope: HOPE VI and the new promise of public housing in American cities, hrsg. v. Cisneros, Henry G.; Engdahl, Lora, S. 15-29. Washington D.C.: The Brookings Institution Press.

Katz, Michael B. 2001. The price of citizenship. Redefining the American welfare state. New York: Henry Holt and Company.

Katz, Michael B.; Stern, Mark J. 2006. One nation divisible. What America was and what it is becoming. New York: Russell Sage Foundation.

Kingsley, Thomas G. 2009 a. »Taking advantage of what we have learned «, in From despair to hope: HOPE VI and the new promise of public housing in American cities, hrsg. v. Cisneros, Henry G.; Engdahl, Lora, S. 263-297. Washington D.C.: The Brookings Institution Press.

Leviathan, 40. Jg., 3/2012 
Kingsley, Thomas G. 2009 b. »Apendix A. Scope and status of the HOPE VI program ", in From despair to hope: HOPE VI and the new promise of public housing in American cities, hrsg. v. Cisneros, Henry G.; Engdahl, Lora, S. 299-306. Washington D.C.: The Brookings Institution Press.

Kingsley, Thomas G.; Pettit, Kathryn L.S. 2007. Concentrated poverty: dynamics of change. The Urban Institute, Neighborhood Change in Urban America, No. 5, August 2007. Washington D.C.: The Urban Institute.

Kneebone, Elizabeth; Garr, Emily 2010. The suburbanization of poverty: trends in metropolitan America, 2000-2008. Metropolitan Policy Program at Brookings, Metropolitan Opportunity Series. Washington D.C.: The Brookings Institution.

Kronauer, Martin 2008. "Ausgrenzung und physisch-sozialer Raum ", in Sozialer Ausschluss und soziale Arbeit. Positionsbestimmungen einer kritischen Theorie und Praxis Sozialer Arbeit. 2., überarbeitete und erweiterte Auflage, hrsg. v. Anhorn, Roland; Bettinger, Frank, S. 181-198. Wiesbaden: VS Verlag für Sozialwissenschaften.

Kronauer, Martin 2010. Exklusion. Die Gefährdung des Sozialen im hoch entwickelten Kapitalismus. 2., aktualisierte und erweiterte Auflage. Frankfurt a. M., New York: Campus.

Levy, Diane K. 2010. The limits of relocation. Employment and family well-being among former Madden/Wells residents. The Urban Institute Program on Neighborhoods and Youth Development, No. 6, August 2010. Washington D.C.: The Urban Institute.

Levy, Diane K.; Gallagher, Megan 2006. HOPE VI and neighborhood revitalization. Final report. Washington D.C.: The Urban Institute.

Ludwig, Jens; Liebman, Jeffrey; Kling, Jeffrey; Duncan, Greg J.; Katz, Lawrence F.; Kessler, Ronald C.; Sanbonmatsu, Lisa 2008. "What can we learn about neighborhood effects from the moving to opportunity experiment? ", in American Journal of Sociology 114, S. 144-188.

Mishel, Lawrence; Bernstein, Jared; Allegretto, Sylvia 2005. The state of working America 2004/ 2005. An economic policy institute book. Ithaca: Cornell University Press.

Mishel, Lawrence; Bernstein, Jared; Shierholz, Heidi 2009. The state of working America 2008/ 2009. An economic policy institute book. Ithaca: Cornell University Press.

Neef, Rainer; Keim, Rolf 2007. »Wir sind keine Sozialen ". Marginalisierung und Ressourcen in deutschen und französischen Problemvierteln. Konstanz: UVK.

Partridge, Mark D.; Rickman, Dan S. 2006. Geography of American poverty. Is there a need for place-based policies? Kalamozoo: W.E. Upjohn Institute for Employment Research.

Popkin, Susan J.; Cunningham, Mary K. 2009. "Has HOPE VI transformed residents' lives? «, in From despair to hope: HOPE VI and the new promise of public housing in American cities, hrsg. v. Cisneros, Henry G.; Engdahl, Lora, S. 191-203. Washington D.C.: The Brookings Institution Press.

Popkin, Susan J.; Leventhal, Tama; Weismann, Gretchen 2008. Girls in the shood: the importance of feeling safe. Three-city study of moving to opportunity. The Urban Institue, Brief No. 1. Washington D.C.: The Urban Institute.

Rosenbaum, Emily; Harris, Laura; Denton, Nancy A. 2003. "New places, new faces: an analysis of neighborhoods and social ties among MTO movers in Chicago ", in Choosing a better life? Evaluating the moving to opportunity social experiment, hrsg. v. Goering, John; Feins, Judith D., S. 275-31. Washington D.C.: Urban Institute Press.

Sampson, Robert J. 2008. »Moving to inequality: neighborhood effects and experiments meet social structure ", in American Journal of Sociology 114, 1, S. 189-231.

Shroder, Mark 2003. "Locational constraint, housing counseling, and successful lease-up «, in Choosing a better life? Evaluating the moving to opportunity social experiment, hrsg. v. Goering, John; Feins, Judith D., S. 59-80. Washington D.C.: Urban Institute Press.

Snel, Erik; Engbersen, Godfried 2009. "Social reconquest as a new policy paradigm: changing urban policies in the city of Rotterdam ", in Between the social and the spatial. Exploring the multiple dimensions of poverty and social exclusion, hrsg. v. De Boyer, Katrien; Dewilde, Caroline; Dierckx, Danielle; Friedrichs, Jürgen, S. 149-166. Farnham, Burlington: Ashgate.

Schwartz, Alex F. 2010. Housing policy in the United States. Second edition. New York, London: Routledge.

The New York Times 2011: Poverty rate soars to highest level since 1993. 14. September 2011, S. A1 und A12. 
Wilson, William Julius 1987. The truly disadvantaged. The inner city, the underclass, and public policy. Chicago, London: Chicago University Press. 
Zusammenfassung: Seit den 1990er Jahren gibt es in den Metropolenregionen der USA erhebliche Veränderungen in der Entwicklung und räumlichen Verteilung der Armut. Darüber hinaus ergriff die amerikanische Regierung politische Initiativen zur Dekonzentration der städtischen Armut. Beides wird hierzulande bislang kaum zur Kenntnis genommen. Der Beitrag gibt einen Überblick über die Entwicklung der Armut in den Metropolenregionen der USA. Sodann stellt er zwei Bundesprogramme vor, die gezielt die räumliche Konzentration städtischer Armut verringern wollen. Er zeigt die Grenzen einer allein auf Mobilität und soziale Mischung setzenden Politik der Armutsbekämpfung auf und zieht Folgerungen für die deutsche und europäische stadtpolitische Diskussion.

Stichworte: Konzentrierte städtische Armut, Nachbarschaftseffekte, Politik der Dekonzentration von Armut

\section{Can we transcend urban poverty? Experiences from the USA}

Summary: Beginning in the 1990 s, considerable changes took place in the development and spatial distribution of poverty in the metropolitan areas of the USA. Also in the $1990 \mathrm{~s}$ the American government initiated policies to reduce the concentration of poverty in urban neighborhoods. Until now both developments have been barely recognized in German scientific and political debate. The article provides an overview of changes in the development and distribution of poverty in the metropolitan areas. It then presents two federal programs targeting concentrated urban poverty. The limitations of policies attempting to fight poverty solely by means of spatial mobility and socially mixing neighborhoods are demonstrated and conclusions for German and European urban policies are suggested.

Keywords: Poverty, USA, neighbourhood effects, Public Housing, concentrated urban poverty, policies of de-concentrating poverty

\section{Autoren}

Hartmut Häußermann war Professor für Stadt- und Regionalsoziologie an der Humboldt-Universität zu Berlin.

Prof. Dr. Martin Kronauer

Hochschule für Wirtschaft und Recht Berlin

Campus Schöneberg

Badensche Straße 52

10825 Berlin 